\title{
AGE CHANGES IN THE ORGANIC FRACTION OF BONE
}

\author{
J. W. Smith, St Andrews, Scotland
}

From the Anatomy Department, St Salvator's College, University of St Andrews

Mammalian bone is a heterogeneous tissue which is divisible at the microscopic level into units or regions of different appearance. The relationship of these units to one another is, in large measure, a reflection of the developmental processes by which the tissue has been formed. Primary bone is laid down either as surface bone (concentric lamellar bone) or as primary osteones, but in many species this is not a permanent tissue. As it ages it undergoes focal resorption and, in the vascular spaces so created, secondary osteones are formed by the centripetal accretion of new bone. Subsequently these secondary osteones are themselves partially resorbed and replaced by new osteones of similar form. Once established, this process of internal resorption and replacement continues throughout life, and the extent of its effects on bone architecture is related to the life span of the species concerned. The bone which surrounds secondary osteones is often described collectively as the interstitial tissue, and its structure exhibits both species and age variations. During the period of growth, and for some time after in many bones, it consists, largely or entirely, of primary bone, either in the form of primary osteones (Figs. 1 and 2) or as surface bone (Fig. 4). However, as age advances the remnants of partially resorbed secondary osteones progressively replace the primary elements, so that eventually these remnants form the greater part, or even the whole, of the interstitial tissue (Fig. 7).

As a result of the nature of the continual resorption/replacement process, it is evident that in any macroscopic volume of bone the constituent microscopic units will often vary greatly in age. Some secondary osteones may be only a few weeks old (Vanderhoeft, Kelly and Peterson 1962; Vincent 1955), while others close to them may have an age of the order of twenty years (Marshall 1960). It is also evident that the average age of a macroscopic volume of bone is seldom equal to the chronological age of the individual; almost invariably it is very much less.

All bone consists of mineral, organic and water fractions, but it is now well known that the proportions in which these fractions are combined vary in adjacent microscopic units, and that these variations are closely associated with the age of these units. As a result of the many investigations which have been carried out in recent years, age changes in the inorganic concentration in bone are now fairly well understood. However, it is evident that in a rigid tissue such as bone, in which volume is constant, variations in the concentration of one fraction involve proportional variations in the concentrations of one or both of the other two. This paper is concerned with the manner in which the organic concentration in bone varies with its age. No attempt has been made to differentiate between the different components of the organic fraction, but since collagen comprises about 95 per cent of the whole (Eastoe 1956) the results are largely related to this substance.

\section{ASSESSMENT OF THE RELATIVE AGE OF MICROSCOPIC REGIONS OF BONE}

It is theoretically possible, by the administration of suitable bone markers, to prepare bone in which the absolute age of any microscopic unit could be determined, but the time range of twenty to thirty years which would be involved makes this impracticable. However, assessment of the relative ages of such units can usually be achieved by consideration of their place in the developmental process, their relationship to the resorption/replacement process, and their spatial relationship to one another. Thus. because of their mode of formation, 
secondary osteones are always younger than the interstitial bone which immediately surrounds them, and this relationship is independent of the nature of the interstitial bone. In interstitial bone itself, such as that illustrated in Figure 8, area F contains Sharpey fibres; it must therefore be a remnant of the original primary bone which occupied the region (Smith 1960b), and is consequently older than the rest of the field which consists of secondary bone. Furthermore, in Figure 8 it is evident from the relationship of the areas A, B and C to one another that A is younger than B which is younger than $\mathrm{C}$. Amongst secondary osteones a complete unit is necessarily younger than one which it has marginally eroded, so that in Figure 7 osteone 6 is younger than osteone 9 , and osteones 1, 2 and 3 are younger than osteone 14. Moreover, an osteone which is still forming and which has a wide vascular canal (Fig. $8 \mathrm{D}$ ) is on the average younger than a completely grown osteone with a definitive vascular canal (Fig. 8 E).

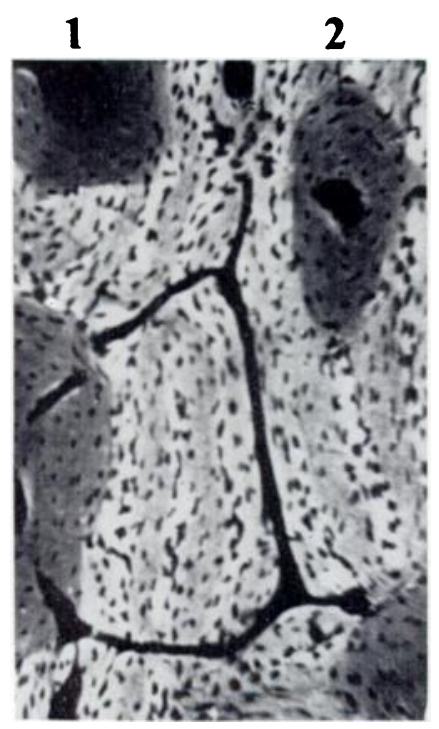

FiG. 1

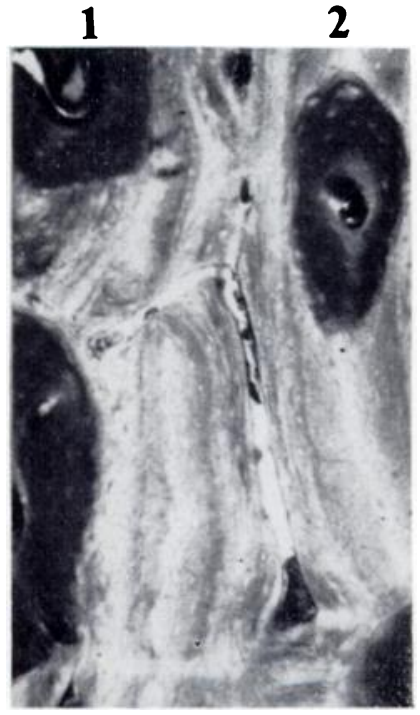

FIG. 2

Transverse ground section of femur of pig. For description see text. Figure 1-Microradiograph. $(\cdot 120$.$) Figure 2-Carbonised$ preparation of same region. $(.120$.

\section{AGE CHANGES IN THE INORGANIC CONCENTRATION IN SECONDARY OSTEONES}

The relative inorganic concentrations in adjacent microscopic regions in bone can be conveniently assessed by contact microradiography of thin ground sections. In the microradiographs illustrated in Figures 1 and 3, regions with a low mineral content appear dark because they are relatively translucent to $\mathrm{x}$-rays. On the other hand, regions in which the mineral content is high are more opaque to $x$-rays, and consequently appear light.

In young secondary osteones which are either in the process of formation or have recently been completed (Fig. 1, osteones 1 and 2) the inorganic concentration is uniformly low and, according to Amprino and Engström (1952), amounts to about 75 per cent of full calcification. The concentration is considerably less in such osteones than in the older interstitial bone tissue which surrounds them. Moreover, this relationship is independent of the nature of the interstitial tissue. Figure 1 shows parts of four young secondary osteones surrounded by a more densely mineralised region of primary osteones, whereas in Figure 3 several young secondary osteones are surrounded by more densely mineralised interstitial tissue, which consists of the remnants of older secondary osteones which have been partially resorbed.

Once the formation of a secondary osteone is complete its inorganic concentration begins to increase. This increase occurs first, and proceeds most rapidly, at the inner margin of the 
osteone, and this distribution produces, initially, the phenomenon described by Harris, Jackson and Jowsey (1962) as edge sclerosis (Fig. 3, osteone A), and subsequently, the characteristic mineral gradient (Fig. 3, osteone B) noted by Amprino and Engström (1952) and by Vincent (1955). At the outer margin of the osteone another-and more localisedincrease of the inorganic concentration occurs in the region of the reversal line, so that in what might be termed a middle-aged osteone (Fig. 3, osteone C) the mineral gradient decreases from the inner margin to the periphery and there rises abruptly to the high level of the reversal line.

Thereafter, as the age of the osteone increases, the inorganic concentration throughout the unit continues to rise asymptotically towards full calcification. Consequently, in old complete osteones (Fig. 3, osteone D), and in the remnants of old osteones which have been partially resorbed (Fig. 3, area E), there is no mineral gradient and the inorganic concentration is uniformly high.

The formation of most secondary osteones is a continuous process which proceeds at a progressively decreasing rate (Lacroix 1960). However, occasionally the process is interrupted by a quiescent period, and in these circumstances the first bone to be laid down when

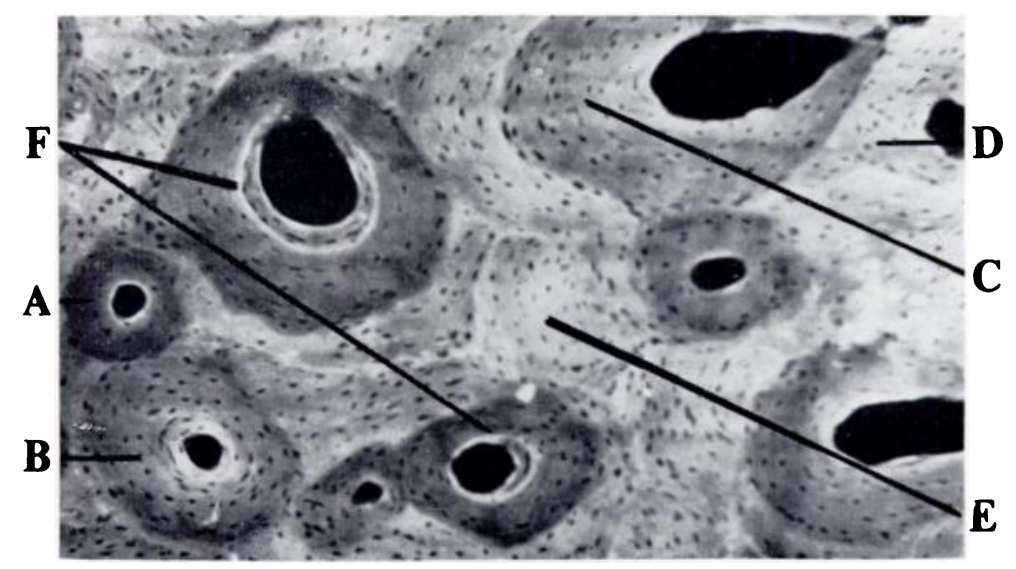

Fig. 3

Microradiograph of ground section of adult human femur. For description see text. $(\times 100$.

osteogenesis restarts rapidly achieves a very high mineral concentration. These narrow zones of highly calcified bone (Fig. 3 F) are known as resting lines, and they are essentially similar in formation and character to the reversal lines discussed above.

\section{AGE CHANGES IN THE ORGANIC CONCENTRATION IN SECONDARY OSTEONES}

In this investigation the relative amounts of organic material in adjacent microscopic regions of bone have been assessed by three methods.

Contact microradiography of decalcified sections-Transverse frozen sections $20 \mu$ thick were prepared from cortical bone decalcified in 5 per cent nitric acid. These were mounted, under a Wratten 1A safelight, on Kodak Experimental Film (V.6028) which had been protected by dipping in a 1 per cent solution of nitrocellulose in ether-ethanol, according to the procedure described by Greulich (1960). The mounted sections were dried overnight in the dark at 25 degrees Centigrade, and were then regarded as consisting of the greater part of the organic fraction of the original bone, with a certain amount of water. The film and section were then exposed in a Phillips Contact Microradiographic Unit No. 5 at an anode potential of two kilovolts for twenty-five milliampere minutes. In these circumstances the smallest wavelength 
in the radiation spectrum is a little over 6 Angström units, and the greatest intensity occurs between 8 and 9 Angström units. At these wavelengths the mass absorption coefficient of the organic material in the section is about 1,000 in gramme centimetre units (Engström and Engfeldt 1953), while that of water is of the same order (Sissons, Jowsey and Stewart 1960), and consequently both components cause an appreciable absorption of the soft radiation. After exposure the section and the protective coating were removed in acetone, and the film was developed, cleared and mounted in balsam.

An example of the microradiographs obtained by this method is illustrated in Figure 4. A number of secondary osteones are surrounded by interstitial tissue which consists of older surface bone. It is evident that the osteones labelled A, B and C are lighter, that is, more opaque to $\mathrm{x}$-rays than the interstitial bone. The greater density of the secondary osteones could be related either to a greater organic concentration in these units or to a greater retention of water by them during the drying of the section. However, because there is certainly more

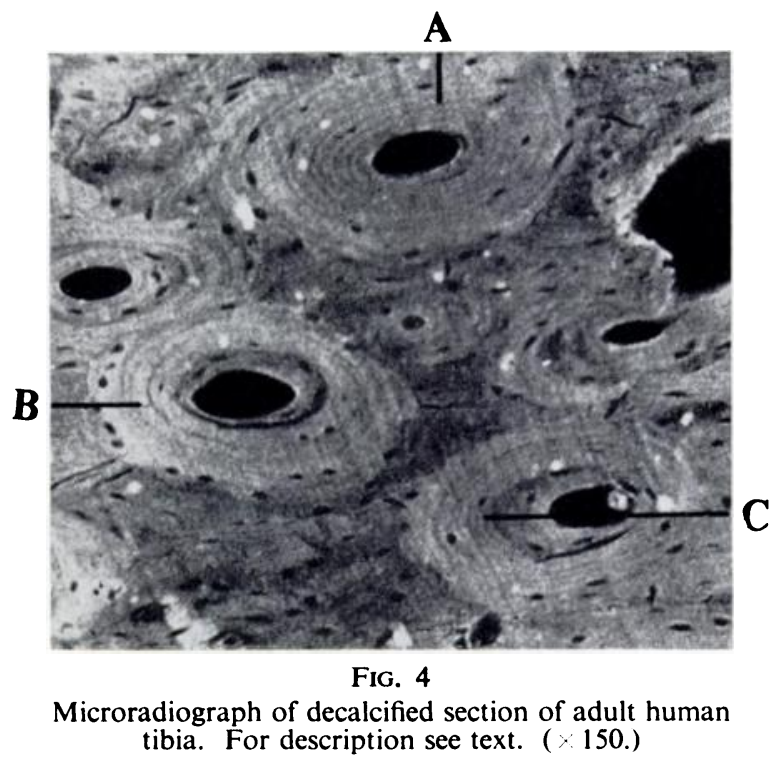

organic material in the section than water, it is considered that the relative radio-opacity of the secondary osteones is due to a greater organic concentration, and this has, in fact, been confirmed by other observations described below.

Two other features in Figure 4 are of interest. First, in osteone A there is distinct evidence of lamellar variation in the concentration of organic material. And secondly, in osteone B, and to a lesser degree in two other osteones in the field, a narrow dark, radio-translucent zone can be seen at a short distance from the inner margin. In this zone the organic concentration is much lower than in the rest of the osteone. It is considered that it represents a resting line, formed after a pause in the process of osteone formation, and that it may therefore be identified with the highly calcified resting lines which were noted in Figure $3 \mathrm{~F}$.

Contact microradiography of "tanned" decalcified sections-The mass absorption coefficient of a material, and consequently its opacity to radiation, is a function of the atomic numbers of its constituent elements. The decalcified sections used in the previous method consist mainly of collagen in which the effective element, nitrogen, has the atomic number 7 . It is evident that if the collagen in these sections could be made to combine selectively with a compound which contained elements of relatively high atomic number, the microradiographic contrast between regions of high and low collagen concentration would be intensified. In fact, as is well known, collagen does combine readily with the salts of several metals, and these 
reactions form the basis of some commercial tanning processes. In the course of the present investigations the best results were obtained with phosphotungstic acid in which tungsten has an atomic number of 74 . The results obtained with mercury and lead salts, although somewhat inferior, were reasonably satisfactory.

The combination of collagen with phosphotungstic acid is prevented by preliminary fixation of the material, whereas it is facilitated by preliminary treatment with strong solutions of certain neutral salts. Consequently, after decalcification of fresh, unfixed, cortical bone in 5 per cent nitric acid, frozen sections were prepared at a thickness of $20 \mu$. These were placed in $3 \mathrm{M}$ calcium chloride overnight, washed in distilled water, and transferred to a 0.5 per cent solution of phosphotungstic acid for one hour. Thereafter the sections were removed individually, dipped in distilled water to remove excess of phosphotungstic acid, and placed between two glass slides between which they were dried overnight at 37 degrees Centigrade. When dry, the sections separated readily from the slides and, being tanned, they were of a

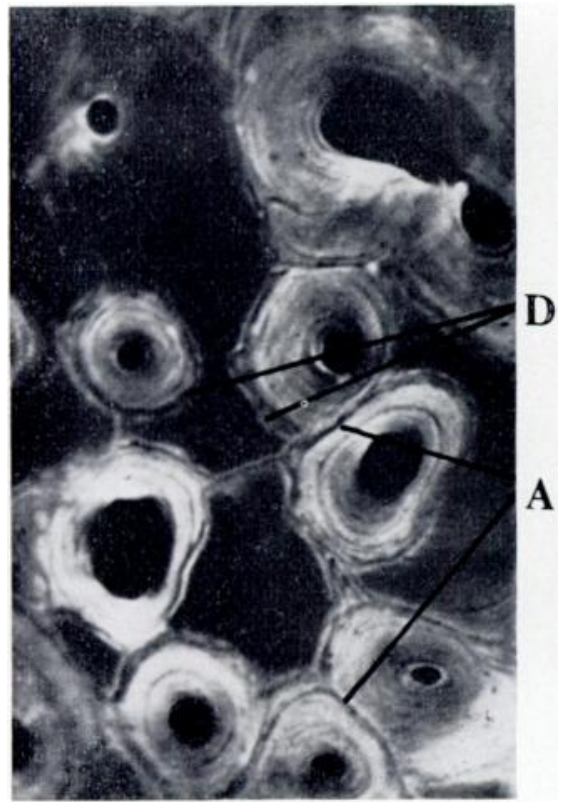

FIG. 5

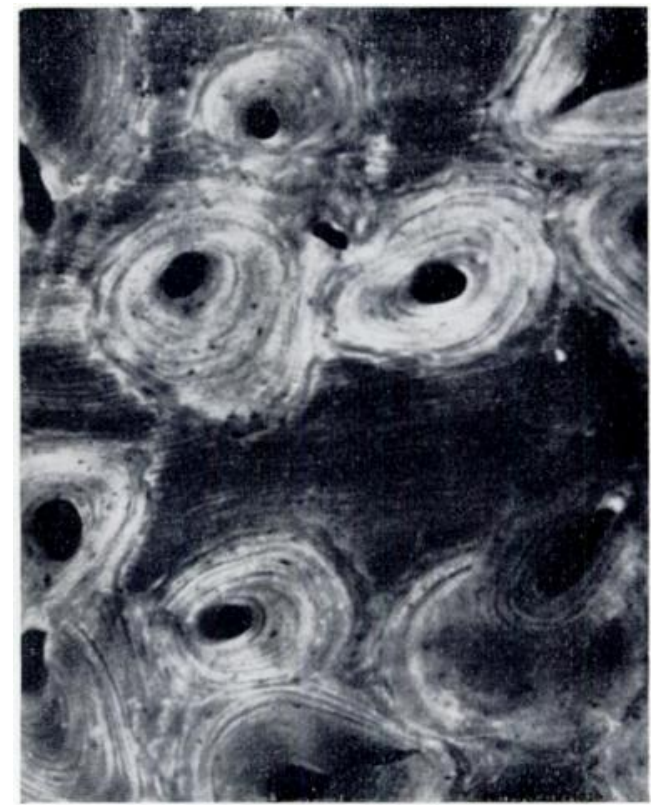

Fig. 6

Microradiographs of decalcified sections treated with phosphotungstic acid. For description see text. Figure 5-Transverse section adult human femur. $(\times 120$.$) Figure 6-$ Transverse section adult human tibia. $(\times 150$.

hard, tough consistency which facilitated subsequent handling. The dry sections were then placed on Kodak Experimental Film and radiographed at an anode potential of five kilovolts for four milliampere minutes. Under these conditions the mass absorption coefficient of the collagen in the original section is only about 20 in gramme centimetre units (Sissons et al. 1960 ) and the only effective absorbing element is tungsten.

Examples of microradiographs prepared by this method are illustrated in Figures 5 and 6. In Figure 5 several secondary osteones are surrounded by interstitial tissue which consists of the remnants of older secondary osteones. It is evident that the relatively young osteones are now much more opaque to $\mathrm{x}$-rays that the interstitial bone: they therefore contain more tungsten, and it is considered that this indicates that they originally contained more collagen. Within some of the osteones there is evidence of lamellar variation of collagen concentration, but this is not so precise as in Figure 4. In the peripheral part of each osteone there is a narrow translucent zone in which the collagen concentration is low. Where two osteones 
abut against one another, as at $\mathrm{A}$ in Figure 5, the zone is readily equated with the highly calcified reversal lines noted in Figure 3. But where an osteone is directly adjacent to interstitial tissue, as at $\mathrm{D}$ in Figure 5, the translucent zone is bordered externally by an opaque zone of similar width, and the explanation of this appearance is not clear.

In Figure 6 the secondary osteones are surrounded by older surface bone, which makes it more directly comparable to Figure 4, but, apart from this distinction, the difference in the collagen concentrations of the osteones and the older interstitial tissue, the suggestion of

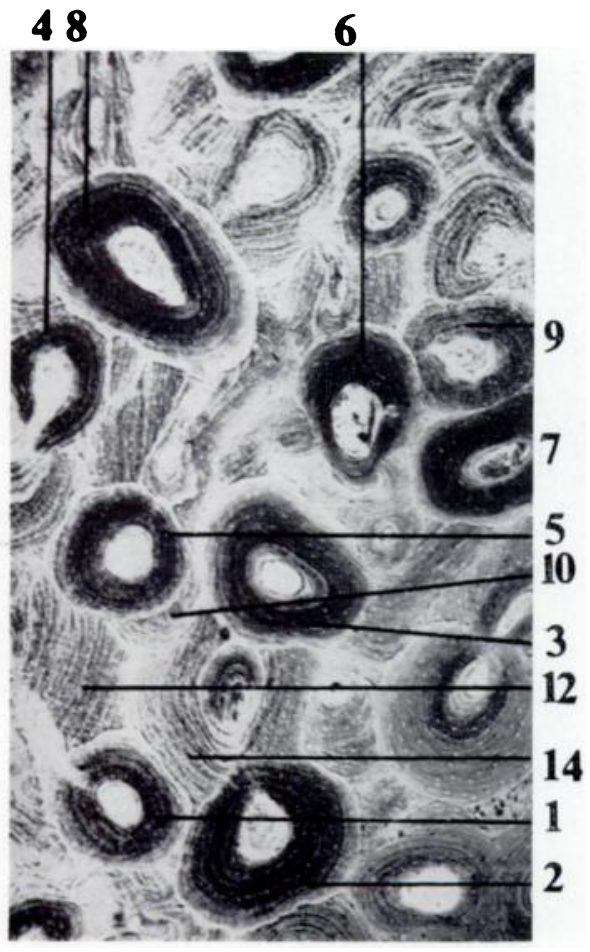

Fic. 7

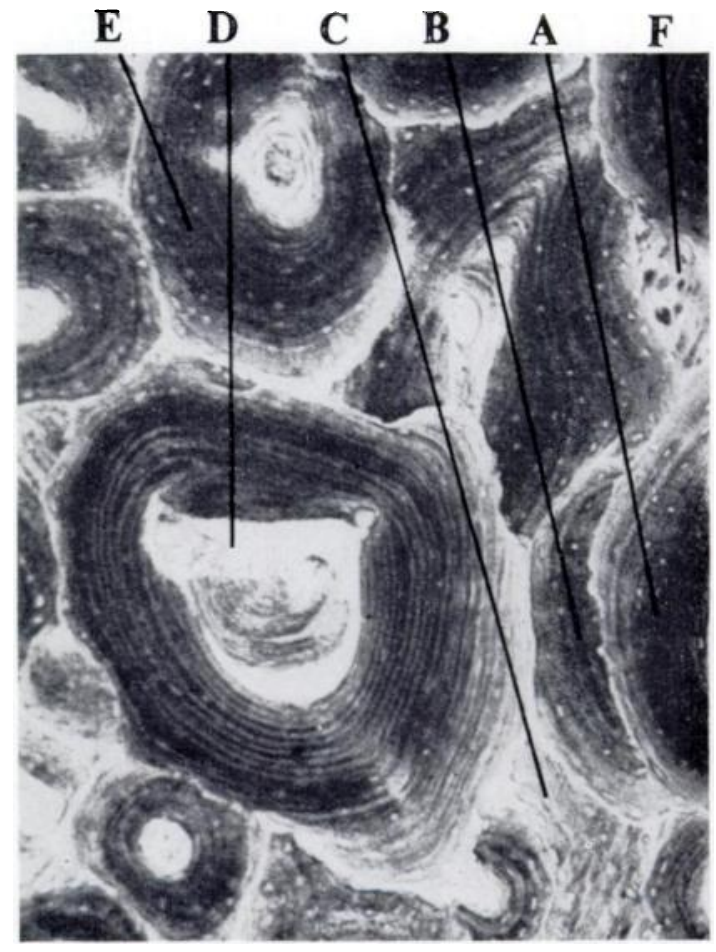

FiG. 8

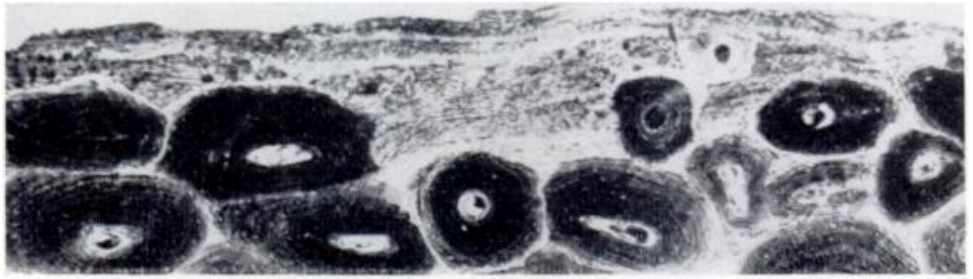

FIG. 9

Carbonised preparations of ground sections of bone. For description see text. Figure 7-Transverse section of adult human femur. (.80.) Figure 8-

Transverse section of adult human femur. ( . 200.) Figure 9-Transverse section, outer table of adult human parietal bone. $(\cdot 80$.)

lamellar variation in collagen concentration, and the low collagen concentration of the reversal lines, are similar to the same features seen in Figure 5.

Carbonisation of undecalcified sections-It is well known that when undecalcified bone is heated it passes through a number of colour changes, becoming in sequence yellow, brown, black and finally white again. These changes in colour cannot be related to any alteration of the mineral fraction of bone, and it is therefore considered that they are due to the reduction of the organic components of the tissue to carbon and certain intermediate degradation products. 
Furthermore, even in the absence of direct proof, it seems a reasonable presumption that, in closely related microscopic units within a small region of bone, the intensity of blackening or the carbon density is a direct function of the original organic concentration. The temperature to which the bone is heated, and the duration of the treatment, are arbitrary matters, but the following procedure has been found to give satisfactory results. Transverse ground sections were prepared at a thickness of $70 \mu$. These were placed between glass slides on an asbestos wire grill, and heated by bunsen burner. When the bone became golden yellow in colour the flame was removed, but, owing to the heat present in the glass slides, the colour of the bone continued to change until, by the time it was cool, it had become dark brown. The sections, at this stage, were very fragile but retained their original form completely. They were placed in xylol, and subsequently mounted in balsam under a coverslip.

The characteristic appearance of such carbonised preparations, viewed by transmitted light, is illustrated in Figure 7. The field contains a number of secondary osteones (1-8) which have comparatively wide vascular canals, and which have not suffered any peripheral erosion by neighbouring resorption cavities. These are regarded as comparatively young regions of bone, and in each of them the carbon density is high. On the other hand, in the same field there are other secondary osteones, which have narrow vascular canals and which have been marginally eroded, and it is considered that these are older regions of bone. Thus osteone 14 in Figure 7 has been marginally eroded by the resorption cavities in which osteones 1,2 and 3 have subsequently formed, and is consequently older than these. Similarly osteone 9 is older than osteone 6 . The remainder of the field consists of the remnants of secondary osteones which have been resorbed centrally by expansion of their own vascular canals. These again are comparatively old regions of bone. For example, area 12 is older than osteones 1 and 5 , and area 10 is older than osteone 5. In all these older regions of secondary bone the carbon density is noticeably less than that in the young complete osteones, and it is considered that this indicates a corresponding difference in the organic concentrations in these regions. Figure 2 shows that a similar relationship exists between the organic concentration in young secondary osteones and that in older primary bone in the form of primary osteones. And similarly, the carbonised preparation in Figure 9 illustrates the high organic concentration in secondary osteones compared with the low organic concentration in the older surface bone within which they have formed.

Figure 8 shows certain additional features of carbonised preparations at a higher magnification. First, each secondary osteone is surrounded by a reversal line in which the carbon density, and consequently the organic concentration, is very low. Secondly, the peripheral part of each osteone, apart from the reversal line, exhibits a carbon density gradient which decreases in a peripheral direction. And thirdly, some osteones, such as the large unit D, show a very distinct lamellar variation in carbon density which is reminiscent of the similar feature noted in osteone $\mathrm{A}$ in Figure 4.

\section{DISCUSSION}

The organic concentration of secondary osteone bone of different ages was studied by Davies and Engström (1954) by interferometry, and as a result of their observations they came to the conclusion that there was no significant change in the amount of organic material in secondary osteones as their age increased. Furthermore, on the basis of this concept, it is now widely held that the age increase which invariably occurs in the mineral concentration of secondary osteone bone takes place entirely at the expense of the water fraction (Robinson and Elliott 1957, Robinson 1960).

On the other hand, the observations presented in this paper indicate that the organic concentration of bone varies appreciably in closely adjacent microscopic units of different age. In young secondary osteones the concentration is very low in the reversal line, but in the rest

VOL. 45 B, NO. 4, NOVEMBER 1963 
of the unit it is high, and there is evidence of a falling gradient in a peripheral direction. The older bone which surrounds secondary osteones may be either primary as in Figures 2, 4, 6 and 9 or secondary as in Figures 5 and 7 , but in both cases its organic concentration is comparatively low. It seems evident, therefore, that the organic concentration of secondary osteone bone is always initially greater than that of the primary bone which it replaces, and that this concentration subsequently decreases as the age of the tissue advances. In contrast the inorganic concentration of secondary osteone bone is initially less than that of the surrounding primary bone, and subsequently increases as the tissue becomes older. It is considered that this association in secondary osteone bone, of an age increase in the inorganic concentration with a simultaneous age decrease in the organic concentration, strongly suggests that the progressive calcification of secondary bone occurs, to some extent at least, at the expense of the organic fraction of the tissue. Moreover, although they have not been demonstrated in this paper, exactly similar age changes occur in the inorganic/organic ratio in primary osteones and surface bone, and consequently it is probable that they represent a process which is common to bone tissue as a whole.

The concept of a decrease in the organic content of bone as its age advances runs counter to the accepted picture of collagen as a material of great stability. Certainly Neuberger and Slack (1953) and Robertson (1952) have shown that collagen has a very low metabolic rate, and Little, Kelly and Courts (1962) have demonstrated that bone collagen may persist, and present a recognisable morphology under the electron microscope, thousands of years after death. However, it is also abundantly clear that in certain situations, and in certain circumstances, collagen can disappear quite rapidly from a tissue or organ. Thus Cameron and Karunaratne (1936) and Morrione (1949) noted that the dense fibrous bands which can be produced in the livers of rats by the administration of carbon tetrachloride, disappear when the toxic material is discontinued. Similarly, Harkness and Harkness (1954) showed that a rapid increase in the collagen content of the rat uterus during pregnancy is followed by a correspondingly rapid decrease during involution. Even in skeletal tissues Fell (1961) demonstrated that, in both cartilage and bone matrix in tissue culture, excess of vitamin A caused a dissolution of collagen, and Little et al. (1962) saw, with the electron microscope, perilacunar areas of degraded collagen in osteoporotic bone. There seems to be no doubt, therefore, that collagen can be removed from living tissues by natural processes. But the nature of these processes, and the manner in which they operate in the specific environment of bone, is not understood.

Two other observations in the present investigation seem to be of interest. In each of the three methods by which the organic fraction of bone has been studied, and particularly in microradiographs of decalcified sections and in carbonised preparations of ground sections, lamellar variations in the organic concentration within osteones are evident. This feature has only been observed in a small percentage of the osteones which have been examined, and it is probable from this incidence that it is related to the Type II fibre pattern described in a previous paper (Smith 1960a). Secondly, both the reversal lines which surround secondary osteones, and the resting lines which are associated with a pause in the process of osteone formation, exhibit a very high inorganic/organic ratio. A similar ratio may also be observed in the woven-fibred plaques which are a feature of some forms of primary bone. These three situations are all associated either with the initiation or the restarting of osteogenesis, and it is hoped to consider them in detail in a subsequent paper.

\section{SUMMARY}

1. The relative concentrations of organic material in adjacent microscopic regions of bone have been studied by three methods, and the results suggest that this concentration varies considerably. 
2. The variations in the organic concentration in bone have been correlated with the age of the bone and its inorganic concentration.

3. It is suggested that the progressive calcification of bone as its age increases, occurs, to some extent, at the expense of the organic fraction of the tissue.

I am greatly indebted to Professor A. Lendrum, Queen's College, Dundee, who suggested to me the use of phosphotungstic acid in the preparation of decalcified sections of bone for microradiography. I am also grateful to Mr James Brown of this department, who prepared the illustrations. The experimental film used in the microradiographic part of the investigation was very kindly supplied by Messrs Kodak Ltd.

\section{REFERENCES}

Amprino, R., and Engström, A. (1952): Studies on X-Ray Absorption and Diffraction of Bone Tissue. Acta Anatomica, 15, 1.

Cameron, G. R., and Karunaratne, W. A. E. (1936): Carbon Tetrachloride Cirrhosis in relation to Liver Regeneration. Journal of Pathology and Bacteriology, 42, 1.

Davies, H. G. and Engström, A. (1954): Interferometric and X-Ray Absorption Studies of Bone Tissue. Experimental Cell Research, 7, 243.

EAstoe, J. E. (1956): The Organic Matrix of Bone. In The Biochemistry and Physiology of Bone, p. 81. Editcd by G. H. Bourne. New York: Academic Press.

EngStröm, A., and Engfeldt, B. (1953): Lamellar Structure of Osteons Demonstrated by Microradiography. Experientia, 9, 19.

Fell, H. B. (1961): Experiments on the Action of Vitamin A on the Ground Substance of Cartilage and Bone in Culture. Journal of Bone and Joint Surgery, 43-B, 180.

Greulich, R. C. (1960): Application of High Resolution Microradiography to Qualitative Experimental Morphology. In X-Ray Microscopy and X-Ray Microanalysis. Edited by A. Engström, V. Cosslett and H. Pattee. Amsterdam: Elsevier.

Harkness, M. L. R., and Harkness, R. D. (1954): The Collagen Content of the Reproductive Tract of the Rat during Pregnancy and Lactation. Journal of Physiology, 123, 492.

Harris, W. H., Jackson, R. H., and Jowsey, J. (1962): The In Vivo Distribution of Tetracyclines in Canine Bone. Journal of Bone and Joint Surgery, 44-A, 1,308.

Lacroix, P. (1960): $\mathrm{Ca}^{45}$ Autoradiography in the Study of Bone Tissue. In Bone as a Tissue. Edited by K. Rodahl, J. T. Nicholson and E. M. Brown, Jun. London: McGraw-Hill Book Company, Inc.

Little, K., Kelly, M., and Courts, A. (1962): Studies on Bone Matrix in Normal and Osteoporotic Bone. Journal of Bone and Joint Surgery, 44-B, 503.

Marshall, J. H. (1960): Microscopic Metabolism of Calcium in Bone. In Bone as a Tissue. Edited by K. Rodahl, J. T. Nicholson and E. M. Brown, Jun. London: McGraw-Hill Book Company, Inc.

Marshall, J. H., White, V. K., and Cohen, J. (1959): Microscopic Metabolism of Calcium in Bone. Radiation Research, 10, 197.

Morrione, T. G. (1949): Factors Influencing Collagen Content in Experimental Cirrhosis. American Journal of Pathology, 25, 273.

Neuberger, A., and Slack, H. G. B. (1953): The Metabolism of Collagen from Liver, Bone, Skin and Tendon in the Normal Rat. Biochenical Journal, 53, 47.

RoBertSON, W. van B. (1952): Influence of Ascorbic Acid on $\mathrm{N}^{15}$ Incorporation into Collagen in Vivo. Journal of Biological Chemistry, 197, 495.

Robinson, R. A. (1960): Chemical Analysis and Electron Microscopy of Bone. In Bone as a Tissue. Edited by K. Rodahl, J. T. Nicholson and E.M. Brown, Jun. London: McGraw-Hill Book Company, Inc.

Robinson, R. A., and Elliott, S. R. (1957): The Water Content of Bone. Journal of Bone and Joint Surgery, 39-A, 167.

Sissons, H. A., Jowsey, J., and Stewart, L. (1960): Quantitative Microradiography of Bone Tissue. In X-Ray Microscopy and $X$-ray Microanalysis. Edited by A. Engström, V. Cosslett and H. Pattee. Amsterdam: Elsevier.

Smirh, J. W. (1960a): The Arrangement of Collagen Fibres in Human Secondary Osteones. Journal of Bone and Joint Surgery, 42-B, 588.

Smith, J. W. (1960b): Collagen Fibre Patterns in Mammalian Bone. Journal of Anatomy, London, 94, 329.

Vanderhoeft, P. J., Kelly, P. J., and Peterson, L. F. A. (1962): Determination of Growth Rates in Canine Bone by means of Tetracycline-labeled Patterns. Laboratory Investigations, 11, 716.

VINCENT, J. (1955): Récherches sur la Constitution de l'Os adulte. Thesis. Université Louvain. Brussels: Éditions Arscia.

VOL. 45 B, NO. 4, NOVEMBER 1963 